\title{
200 mL Rapid Drink Challenge During High- resolution Manometry Best Predicts Objective Esophagogastric Junction Obstruction and Correlates With Symptom Severity
}

\author{
Philip Woodland, Shirley Gabieta-Sonmez, Julieta Arguero, Joanne Ooi, Kenichiro Nakagawa, Esteban Glasinovic, Etsuro Yazaki, \\ and Daniel Sifrim*
}

Barts and the London School of Medicine and Dentistry, Queen Mary University of London, London, UK

\begin{abstract}
Background/Aims
Single swallow integrated relaxation pressure (IRP) on high-resolution manometry (HRM) does not always accurately predict esophagogastric outflow obstruction on timed barium esophagogram (TBE). Furthermore, neither single swallow IRP or TBE is reliable in predicting symptoms, particularly after treatment with dilatation or myotomy. A $200 \mathrm{~mL}$ rapid drink challenge (RDC) has been proposed as an adjunctive test during HRM. This serves as a "stress-test" to the esophagogastric junction, and may yield clinically useful parameters. We aim to assess HRM parameters during RDC, and their ability to predict outflow obstruction on TBE in patients with dysphagia, and to correlate with symptoms in patients' achalasia.
\end{abstract}

\section{Methods}

Thirty patients with dysphagia were recruited. All underwent standard single swallow HRM analysis, $200 \mathrm{~mL}$ RDC, then TBE. RDC parameters, including esophagogastric pressure gradient, IRP, and RDC duration were evaluated. Multiple regression analysis was performed to assess the best predictive parameter for obstruction on TBE. A further 21 patients with achalasia were evaluated with Eckhardt score, single swallow HRM, RDC, and TBE. Parameter correlation with Eckhardt score was evaluated.

\section{Results}

Mean IRP during RDC was the best HRM parameter at predicting outflow obstruction on TBE. This performed much better in untreated patients (sensitivity $100 \%$ and specificity $85.5 \%$ ) than in previously treated patients (sensitivity $50 \%$ and specificity $66 \%$ ). In patients with achalasia, mean IRP during RDC was the only parameter that correlated with symptom score.

\section{Conclusion}

Mean IRP during RDC appears to be a clinically useful "stress test" to the esophagogastric junction during HRM.

(J Neurogastroenterol Motil 2018;24:410-414)

Key Words

Achalasia; Dysphagia; Esophageal manometry; High resolution

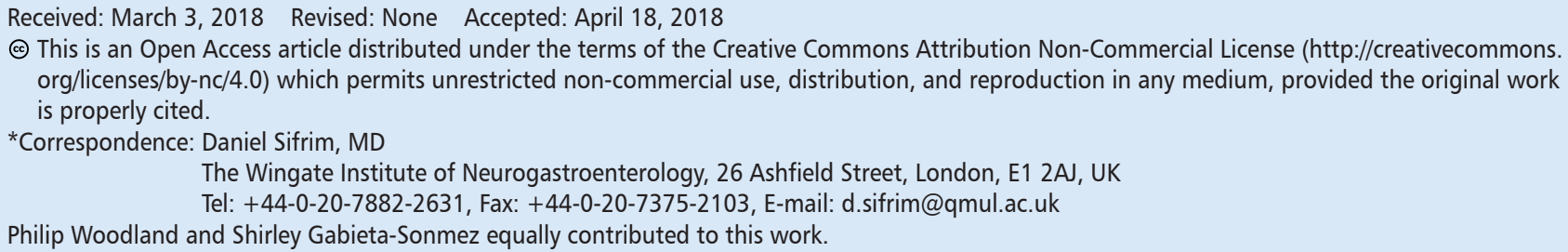




\section{Introduction}

High-resolution manometry (HRM) is a commonly used tool for assessing dysphagia in patients with normal upper gastrointestinal endoscopy. Such topographical studies allow detailed analysis of esophageal peristalsis and smooth muscle contraction and relaxation. A failure of relaxation of the lower esophageal sphincter (LES) is a clinically important cause of dysphagia. It is usually seen in 2 settings: achalasia and esophagogastric junction (EGJ) outflow obstruction. These are classified in the Chicago classification of esophageal motility disorders v $3.0^{1}$ at the top of the hierarchical assessment of esophageal motility by HRM due to their high association with esophageal symptoms.

Whilst HRM analysis according to the Chicago classification has great utility in assessing esophageal motor disorders, it does not always give clear indication as to the degree of any EGJ obstruction, nor do findings necessarily correlate well with patient symptoms. ${ }^{2}$

Tools are available to give more information about esophagogastric junction opening and bolus flow. The combination of HRM with impedance can help understand the relationship between LES relaxation and bolus flow, ${ }^{3}$ and impedance planimetry with Endoflip informs about LES compliance and opening. ${ }^{4}$ One of the most useful and frequently implemented tests is the timed barium esophagogram (TBE). This allows an objective assessment of flow across the EGJ, and has been used extensively in achalasia. Whilst TBE is likely to be helpful in the untreated achalasia patient where sphincter opening is poor, it may be less helpful in the treated patient. Whilst retained barium post treatment appears to predict long term treatment failure, it does not necessarily correlate with symptoms. ${ }^{5}$ Furthermore, a slow challenge of liquid barium may flow through a treated sphincter, even if the degree of opening is insufficient for symptom relief in every day conditions.

Recent studies have shown that a $200 \mathrm{~mL}$ rapid drink challenge (RDC) during HRM can help in the diagnosis of esophageal motility disorders (Fig. 1). ${ }^{6,7}$ During the RDC there should be LES relaxation and inhibition of esophageal body contractions. Also, during the RDC there is a build-up of intra-esophageal pressure due to volume-loading, and this pressure is higher if there is obstruction to esophageal outflow at the EGJ.

A TBE is a high volume, slow speed challenge to the EGJ, and a single water swallow is a low volume, high speed challenge. The RDC allows a high volume, high speed "stress test" to LES opening, and may be able to provide more clinical relevance.

We hypothesised that the RDC would be able to diagnose obstruction at the EGJ in patients with dysphagia, and may better predict symptomatology in patients with achalasia (treated or untreated) than other HRM or TBE metrics.

\section{Materials and Methods}

To test the ability of RDC to predict outflow obstruction on TBE we prospectively investigated 30 patients attending the Royal London Hospital upper gastrointestinal physiology unit with dysphagia. All patients underwent high-resolution esophageal manometry (Manoscan, Medtronic, Minneapolis, MN, USA) according

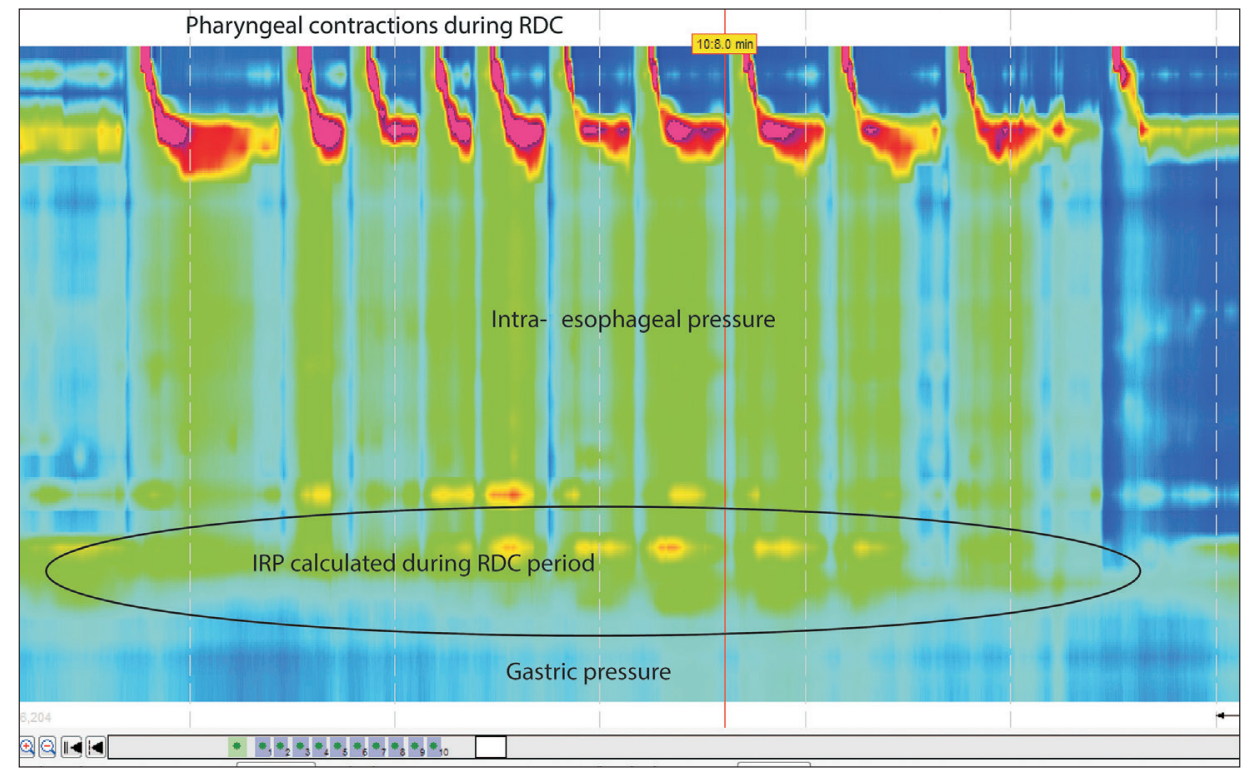

Figure 1. Example of a rapid drink challenge (RDC) on manometry. IRP, integrated relaxation pressure. 
Table 1. High-resolution Manometry Predictors of Esophagogastric Junction Obstruction on Timed Barium Esophagogram

\begin{tabular}{|c|c|c|c|c|c|}
\hline Parameters (cut-off value) & $\begin{array}{c}\text { Sensitivity } \\
(\%)\end{array}$ & $\begin{array}{c}\text { Specificity } \\
(\%)\end{array}$ & $\mathrm{LR}+$ & LR- & AUC \\
\hline Esophageal pressure ( $\geq 27.2 \mathrm{mmHg})$ & 70 & 70 & 2.3 & 0.4 & 0.74 \\
\hline Gastric pressure $(\geq 17.9 \mathrm{mmHg})$ & 40 & 50 & 0.8 & 1.2 & 0.49 \\
\hline Esophageal-gastric gradient ( $\geq 7.7 \mathrm{mmHg}$ ) & 70 & 80 & 3.5 & 0.4 & 0.72 \\
\hline $\operatorname{IRP} 200 \mathrm{~mL}(\geq 17 \mathrm{mmHg})$ & 75 & 90 & 7.5 & 0.28 & 0.81 \\
\hline $200 \mathrm{~mL}$ duration $(\geq 21.8 \mathrm{sec})$ & 60 & 50 & 1.2 & 0.8 & 0.58 \\
\hline Number of columns greater than $20 \mathrm{mmHg}(\geq 12)$ & 40 & 50 & 0.8 & 1.2 & 0.54 \\
\hline Sum of time greater than $20 \mathrm{mmHg}(\geq 16.7 \mathrm{sec})$ & 65 & 80 & 3.2 & 0.43 & 0.68 \\
\hline Percentage of time with pressure greater than $20 \mathrm{mmHg}(\geq 0.74)$ & 70 & 80 & 3.5 & 0.37 & 0.76 \\
\hline
\end{tabular}

$\mathrm{LR}+$, positive likelihood ratio; LR-, negative likelyhood ratio; AUC, area under the curve; IRP, integrated relaxation pressure.

Table 2. Parameters in Untreated Patients Only

\begin{tabular}{lccccc}
\hline \multicolumn{1}{c}{ Parameters (cut-off value) } & Sensitivity $(\%)$ & Specificity $(\%)$ & LR+ & LR- & AUC \\
\hline IRP 200 $\mathrm{mL}(\geq 17 \mathrm{mmHg})$ & 100 & 86 & 7 & 0 & 0.96 \\
IRP single swallows & 75 & 71 & 2.6 & 0.35 & 0.64 \\
\hline
\end{tabular}

LR+, positive likelihood ratio; LR-, negative likelyhood ratio; AUC, area under the curve; IRP, integrated relaxation pressure.

to our standard protocol with ten $5 \mathrm{~mL}$ water swallows. All patients then underwent RDC with $200 \mathrm{~mL}$ water to be drank as quickly as possible without a break. This was then followed immediately by TBE.

Analysis of the HRM followed the Chicago classification v3.0. ${ }^{1}$ Analysis of the RDC was done according to that described by Marin and Serra. ${ }^{7}$ The following parameters were recorded during the RDC: intra-esophageal pressure, intragastric pressure, esophagogastric pressure gradient, LES integrated relaxation pressure (IRP), number of intra-esophageal pressure columns $>20$ $\mathrm{mmHg}$, sum of time with intra-esophageal pressure $>20 \mathrm{mmHg}$, and percentage of time with intra-esophageal pressure $>20$ $\mathrm{mmHg}$.

The TBE was performed immediately after the HRM study by swallow of $200 \mathrm{~mL}$ barium sulphate with x-ray assessment at 1,3 , and 5 minutes. Residual esophageal barium column height in $\mathrm{mm}$ was measured at these timepoints. Esophagogastric outflow obstruction was defined by the presence of any persistent barium column on TBE at 5 minutes. Multiple regression analysis was performed to test correlation of all RDC parameters with the presence of outflow obstruction on TBE, and ROC curve analyses performed.

In the second aspect to the study, we tested the ability of RDC parameters to predict symptoms severity in patients with known untreated or treated achalasia. Twenty-one patients with achalasia were evaluated with Eckhardt score, taken at the time of HRM and TBE study. They also underwent standard HRM protocol, $\mathrm{RDC}$ and TBE (with parameters tested as above). Linear regression and correlation between RDC parameters and Eckhardt score was evaluated.

\section{Results}

In part one of the study, mean age of the subjects was 56 years (range 17-75, 17 female). HRM diagnosis in patients with dysphagia was normal $(\mathrm{n}=3)$, achalasia type I $(\mathrm{n}=4)$, achalasia type II ( $\mathrm{n}$ $=18$ ), absent contractility $(\mathrm{n}=1)$, and diffuse esophageal spasm ( $\mathrm{n}$ $=3$ ). Nineteen patients were untreated, 11 had undergone previous treatment for dysphagia ( 5 pneumatic dilatation and 6 Heller's myotomy).

Analysis established that the HRM parameter that best predicted EGJ obstruction on TBE was the IRP during the RDC. On ROC analysis, a cutoff of $17 \mathrm{mmHg}$ had a sensitivity of $75 \%$ and specificity $90 \%$ (Table 1). One patient in our study had a residual barium column despite normal single swallow IRP, and this patient was detected by IRP during RDC.

When only the 19 untreated patients were considered, sensitivity and specificity of IRP during RDC were extremely good (sensitivity $100 \%$ and specificity $86 \%$; Table 2 ). However, this is contrasted with poor performance in previously treated subjects (sensitivity $50 \%$ and specificity $66 \%$ ).

In the second aspect of the study mean age of patients with 
Table 3. Correlations Between High-resolution Manometry Parameters and Eckhardt Score

\begin{tabular}{lcc}
\hline \multicolumn{1}{c}{ Parameter } & $r$ & $P$-value \\
\hline Single swallow IRP & 0.12 & 0.612 \\
200 mL rapid drink challenge IRP & 0.56 & 0.009 \\
200 mL rapid drink challenge & 0.40 & 0.072 \\
esophagogastric gradient & & \\
Timed barium column 1 minute & 0.27 & 0.218 \\
Timed barium column 5 minutes & 0.32 & 0.150
\end{tabular}

IRP, integrated relaxation pressure.

known outflow obstruction was 55 years (range 17-75, 7 female). Nineteen patients had type II achalasia and 2 had type I achalasia.

Mean Eckhardt score was 5.5 (range 2-11). Mean single swallow IRP was $23.7 \mathrm{mmHg}$, mean IRP during RDC was 28.1 $\mathrm{mmHg}$ (range 2.5-70.0 $\mathrm{mmHg}$ ), mean oesophagogastric pressure gradient was $17.3 \mathrm{mmHg}$, and mean barium column height during TBE was $66 \mathrm{~mm}$ and $28 \mathrm{~mm}$ at 1 minute and 5 minutes, respectively. Neither single swallow IRP nor TBE parameters correlated with symptom score. The only parameter that correlated with symptom score was IRP during RDC $(r=0.56$ and $P=0.009)$ (Table 3 and Fig. 2).

\section{Discussion}

The $200 \mathrm{~mL} \mathrm{RDC}$ is a useful adjunct in assessment of esophageal motility disorders, and has been described in recent clinical papers. ${ }^{6,7}$ This study further describes the value of the RDC, particularly in relation to patients with dysphagia and achalasia.

This study demonstrated: (1) in untreated, but not treated patients with dysphagia the mean IRP during RDC is the HRM parameter that best correlates with findings on the TBE and (2) the mean IRP during RDC out-performed other HRM or TBE parameters in predicting symptom severity in patients with known achalasia, whether untreated or treated.

It is a well-documented finding that HRM and barium characteristics do not always correlate well to symptoms in patients with EGJ outflow obstruction (the most frequent group of whom have achalasia). ${ }^{2,8,9}$ Part of this may be due to the inability of eg, HRM to investigate beyond circular muscle contraction or TBE to assess intra-esophageal pressurisation, but a further factor may be the unphysiological nature of the tests. The standard ten $5 \mathrm{~mL}$ water swallows during HRM are valuable, and have allowed the application of standardised diagnostic criteria in their interpretation. ${ }^{1}$ However, there are limitations in confining studies to single swallow

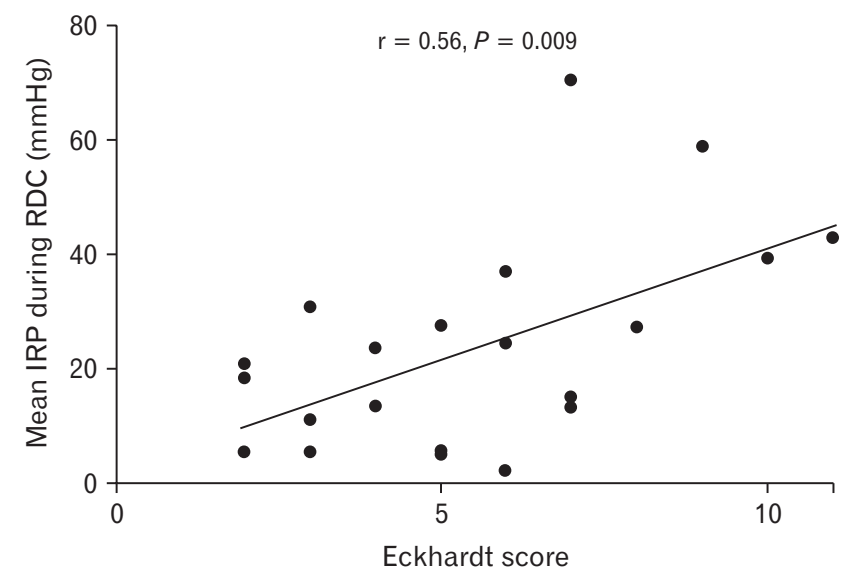

Figure 2. Correlation between Eckhardt score and mean integrated relaxation pressure (IRP) during rapid drink challenge (RDC).

parameters. In particular, they are not representative of swallowing in everyday conditions. In contrast, application of solid meal swallows to an HRM protocol may allow for "unmasking" of motility disorders not seen on single swallows, ${ }^{10}$ but can be cumbersome to perform and interpret. The RDC may offer a compromise between the 2 extremes, and may be particularly useful in understanding gastroesophageal junction characteristics. It offers a standardisable and measureable "stress test" to the EGJ that may be clinically relevant.

In untreated patients with dysphagia, the IRP during RDC was the best manometric parameter to predict hold up on TBE. The IRP is the parameter that should most represent the resistance to esophageal emptying offered by the EGJ, and the IRP during the RDC "stress-test" outperforms single swallow IRP. This is demonstrated by its excellent sensitivity and specificity in predicting outflow obstruction as seen on TBE. In treated patients, the correlation between IRP during RDC and hold up on TBE does not perform as well. The study did not assess why this is the case. It is possible that the RDC becomes less reliable after treatment, but it may be more likely that the TBE becomes less reliable than the RDC after treatment to the LES with dilatation or myotomy. The sphincter does not have to open much to allow a slowly drunk barium drink to pass through the gastroesophageal junction. The high-volume, high-speed RDC is likely to require a greater degree of opening to prevent intrabolus pressure build up within the esophagus (and hence a higher IRP), and so could conceivably be more sensitive for lesser degrees of EGJ obstruction. Therefore, in a treated patient, the absence of a column does not necessarily exclude moderate obstruction. In contrast, the presence of an increased IRP during RDC (even in the absence of a column) might imply persis- 
tent obstruction. This may have re-treatment implications.

Assessment of symptom correlation in the untreated and treated situation may give us some indication of the relative clinical relevance of the parameters. The IRP during RDC outperformed other HRM parameters and TBE parameters in predicting symptoms in untreated and treated achalasia. This suggests that this parameter is clinically relevant, and perhaps confirms the increased sensitivity in detecting clinically relevant obstruction in the previously treated patient.

Single $5 \mathrm{~mL}$ water swallows in HRM assess a high speed but only very low volume challenge to the oesophagus and EGJ. The TBE uses a much more physiological volume (200-250 mL), but assess this over a prolonged time period ( 5 minutes). Our finding that IRP during RDC is able to better predict symptom severity than single swallow HRM parameters or TBE findings is interesting. It is likely to be because the RDC presents a high volume, high speed stress test to the EGJ that is more representative of symptomatic swallowing than found during $5 \mathrm{~mL}$ swallows or the TBE.

This study has some limitations. It did not include measures of EGJ distensibility (eg, Endoflip) ${ }^{11}$ that could have helped better understand the relationship between EGJ distensibility and RDC parameters. We also did not evaluate the ability of RDC parameters to predict outcome to treatment. In the symptom evaluation, the Eckhardt score was used. This is well validated in the untreated situation, but was not devised to evaluate the treated situation. However, there is no specific tool to evaluate symptomatic treatment response in achalasia.

In summary, the mean IRP during RDC appears to have important clinical characteristics. In the untreated patient, it is excellent at demonstrating EGJ obstruction as would be revealed on TBE. This means it can be used as an adjunctive test to reveal outflow obstruction in patients with dysphagia.

In the treated patient, it is thus far uncertain whether the mean IRP during RDC predicts need for retreatment, and further outcome studies are required to evaluate this. However, there is some encouragement that this parameter is clinically useful in both treated and untreated patients, due to the better correlation with symptoms compared to other parameters, such as HRM or TBE.

\section{Financial support: None.}

\section{Conflicts of interest: None.}

Author contributions: Philip Woodland, Shirley Gabieta-Sonmez, Julieta Arguero, Joanne Ooi, Kenichiro Nakagawa, Esteban Glasinovic, and Etsuro Yazaki recruited patients and performed the studies; Philip Woodland, Shirley Gabieta-Sonmez, Julieta Arguero, and Esteban Glasinovic performed analysis; Philip Woodland, Shirley Gabieta-Sonmez, and Daniel Sifrim designed the study; and Philip Woodland and Daniel Sifrim wrote the paper.

\section{References}

1. Kahrilas PJ, Bredenoord AJ, Fox M, et al. The Chicago Classification of esophageal motility disorders, v3.0. Neurogastroenterol Motil 2015;27:160-174.

2. Xiao Y, Kahrilas PJ, Nicodeme F, Lin Z, Roman S, Pandolfino JE. Lack of correlation between HRM metrics and symptoms during the manometric protocol. Am J Gastroenterol 2014;109:521-526.

3. Lin Z, Carlson DA, Dykstra K, et al. High-resolution impedance manometry measurement of bolus flow time in achalasia and its correlation with dysphagia. Neurogastroenterol Motil 2015;27:1232-1238.

4. Kwiatek MA, Pandolfino JE, Hirano I, Kahrilas PJ. Esophagogastric junction distensibility assessed with an endoscopic functional luminal imaging probe (EndoFLIP). Gastrointestinal endoscopy 2010;72:272-278.

5. Vaezi MF, Baker ME, Achkar E, Richter JE. Timed barium oesophagram: better predictor of long term success after pneumatic dilation in achalasia than symptom assessment. Gut 2002;50:765-770.

6. Ang D, Hollenstein M, Misselwitz B, et al. Rapid drink challenge in high-resolution manometry: an adjunctive test for detection of esophageal motility disorders. Neurogastroenterol Motil 2017;29:e12902.

7. Marin I, Serra J. Patterns of esophageal pressure responses to a rapid drink challenge test in patients with esophageal motility disorders. Neurogastroenterol Motil 2016;28:543-553

8. Blam ME, Delfyett W, Levine MS, Metz DC, Katzka DA. Achalasia: a disease of varied and subtle symptoms that do not correlate with radiographic findings. Am J Gastroenterol 2002;97:1916-1923.

9. Ates F, Vaezi MF. Approach to the patient with presumed extraoesophageal GERD. Best Pract Res Clin Gastroenterol 2013;27:415-431.

10. Ang D, Misselwitz B, Hollenstein M, et al. Diagnostic yield of highresolution manometry with a solid test meal for clinically relevant, symptomatic oesophageal motility disorders: serial diagnostic study. Lancet Gastroenterol Hepatol 2017;2:654-661.

11. Rohof WO, Hirsch DP, Kessing BF, Boeckxstaens GE. Efficacy of treatment for patients with achalasia depends on the distensibility of the esophagogastric junction. Gastroenterology 2012;143:328-335. 